\title{
Insights into the clinical effectiveness of whitening products. Dentist-supervised-at-home bleaching product (Opalescence PF). Whitening of darker teeth in contrast to the effects on relatively whiter teeth
}

\begin{abstract}
This study evaluated the success of the whitening of darker teeth by comparing the results of a tooth whitening programme in two groups, subjects with darker (A2 and darker) teeth and patients with whiter teeth. Opalescence PF 10\% was applied for 14 days (nightly for 6-8 hours) and the colour changes followed over a six month period. The overall effect $\left(\triangle \mathrm{E}^{*}{ }_{\mathrm{ab}}\right)$ showed a much higher improvement $(p<1 \%)$ in the whitening of darker teeth $(\sim 40 \%)$ relative to teeth which at the outset had been whiter than A2. Overall $\left(\Delta \mathrm{E}^{\star}{ }_{\mathrm{ab}}\right)$ a deterioration of about $19 \%$ for darker teeth and $\sim 9 \%$ for whiter teeth was seen after six months, but a significant improvement in colour was still evident. Conclusion: The effect of Opalescence PF 10\% treatment was significantly better in all components $\left(L^{*}, a^{*}, b^{*}\right.$ and $\Delta \mathrm{E}^{*}{ }_{\mathrm{ab}}$ ) for $\mathrm{A} 2$ and darker teeth relative to whiter teeth. Despite a significant colour relapse after a six-month period, there was still an improvement compared with before treatment. Thus, it can be expected that bleaching treatment of darker teeth would result in more aesthetic observable colour changes. The significant loss of the whitening effect found after six months may suggest the recommendation to re-bleach.
\end{abstract}

\section{INTRODUCTION}

Tooth whitening in cosmetic dentistry has continuously evolved in the last decade. Film stars took the leading role

1. Sias R Grobler: BSc, BSc (Hons), MSc (Phys Chem), PhD (Dent), DSc (Anal Chem). Oral and Dental Research Institute, Faculty of Dentistry, University of the Western Cape, Tygerberg, Cape Town, South Africa.

2. Yusuf Osman: $B C h D, M C h D$, Hons $B B A, M B A, P G D H M$. Oral and Dental Research Institute, Faculty of Dentistry, University of the Western Cape, Tygerberg, Cape Town, South Africa.

\section{Corresponding author}

Sias R Grobler:

Oral and Dental Research Institute, Faculty of Dentistry, University of the Western Cape, Private Bag X1, Tygerberg 7505, Cape Town, South Africa. E-mail: srgrobler@uwc.ac.za in whitening their teeth on a regular basis, an example which had a copy-cat impact on the general public, leading to increasing demand.

The different ways in which teeth can be whitened include dentist-supervised home bleaching (nightguard vital bleaching), in-office or power bleaching, a combination of in-office and take-home bleaching, as well as over-thecounter whitening products, for use at home..$^{1-3}$

Nowadays, tooth bleaching is mainly done with different peroxides. Hydrogen peroxide forms free radicals like hydroxyl and perhydroxyl, together with, superoxide anions(unstable reactive oxygen molecules which are transformed into oxygen and hydrogen peroxide anions). ${ }^{4}$ Today it is known that tooth discolouration varies in appearance, aetiology, localization, severity and degree of adsorption to tooth structure, which last can be intrinsic, extrinsic or a combination. Intrinsic discolouration is mainly caused by the incorporation of chromatogenic material in enamel and dentine, exposure to high fluoride levels from different sources, tetracycline intake and other factors. ${ }^{1-3}$ Extrinsic stain (as the word implies) comes mainly from the consumption of all kinds of foodstuff with different colouring pigments like carrots, wine (mainly red wine), coffee, tea, etc.

Given the different degrees of darkening, it may be enquired whether darker teeth would whiten more than whiter teeth? The present investigation sought to clarify the question by means of a clinical study.

\section{METHODS AND MATERIALS}

Students with two sound maxillary teeth (tooth 11 and 21) at the Dental Faculty were included in the study (Registered Project \#: UW, 10/3/29). These students were medically healthy, and were not on any treatment. None had undergone any whitening treatment previously. The treatment process followed was as outlined by the Opalescence PF 10\% company. Bleaching was done 
every night (for about 6-8 hours) over a 14 day period. A spectrophotometer (Model: CM-2600d, Konica Minolta Sensing, Inc., Japan) was used to measure the colour of teeth before treatment (base-line), after 14 days and after six months.

\section{RESULTS AND DISCUSSION}

The spectrophotometer enables the quantification of colours by measuring them numerically in a three dimensional colour space $\left(L^{*} a^{*} b^{*}\right){ }^{5}$ Total colour includes three components which are defined as $a^{*}, b^{*}$ and $L^{*}$ (Figure 1).

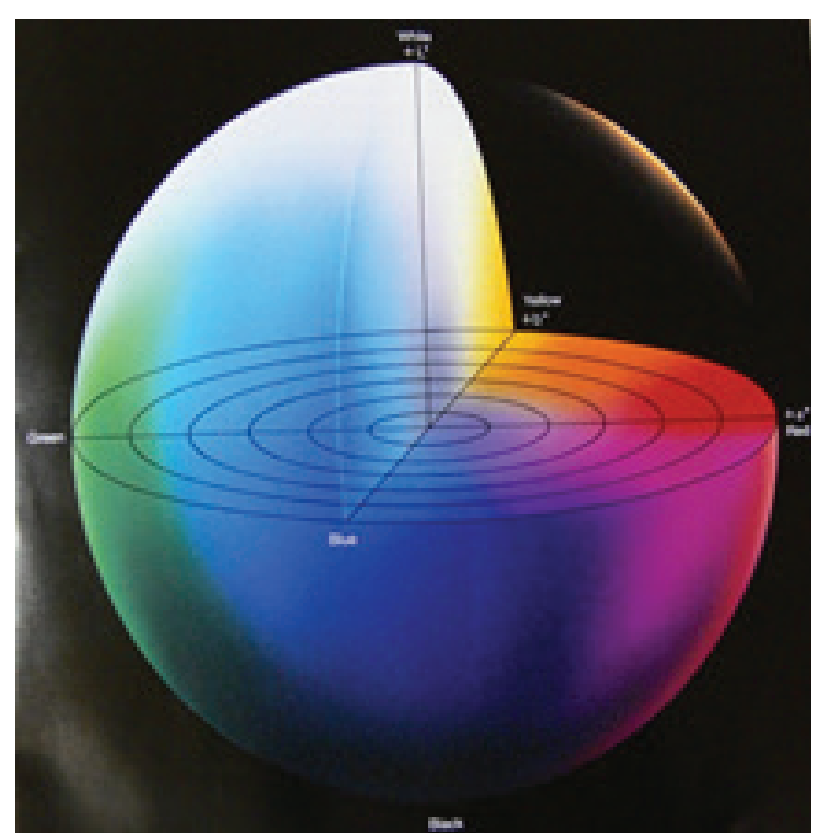

Figure 1: The three- dimensional colour diagram. ${ }^{5}$

Previous contributions (Insights 1 and Insights 2$)^{6}$ have reported on the overall positive effect of Opalescence PF $10 \%$ on the whitening of teeth and on the less demonstrable whitening achieved by an LED treatment. ${ }^{7}$ This section investigated what can be expected when darker teeth (group One) were treated in comparison with the effects on whiter teeth (group Two).

For both groups, all three components $\left(L^{\star}, a^{*}\right.$ and $\left.b^{*}\right)$ showed differences (improvements) between baseline values and the values obtained after treatment (14 days later), and between base-line and after six months $(p<0.01$; Wilcoxon Signed Rank Sum Test). For darker teeth after six months: the decrease in $L^{*}$ (less lighter/ brighter) from base-line was a low $\sim 8 \%$, the value for $\mathrm{a}^{*}$ did not change significantly while the $b^{*}$ value became more yellow, a relapse of about $20 \%$. The deterioration in the overall colour was $19 \%$ after six months. However, it is clear that there still remained a significant improvement in colour compared with before treatment.

The question now is whether these bleaching effects would be visible to the human eye and therefore warranted. If we accept that an overall colour change of one or more units in $\Delta \mathrm{E}^{\star}{ }_{\mathrm{ab}}$ would be visible to the human eye ${ }^{8}$ it becomes clear that for both whiter and darker teeth, the bleaching effect would be clearly visible after the 14 day treatment as well as after a six month period, with $\Delta \mathrm{E}^{\star}{ }_{\mathrm{ab}}$ values of 5.2 and 4.2 for darker teeth, and 3.2 and 3.0 for whiter teeth.

\section{CONCLUSION}

The effect of Opalescence PF 10\% treatment was significantly higher in all components $\left(L^{*}, a^{*}, b^{*}\right.$ and $\left.\Delta E^{*}{ }_{a b}\right)$ for $\mathrm{A} 2$ and darker teeth relative to changes seen in whiter teeth. The response was more visible for darker teeth. A significant colour relapse after a six-month period was found for both groups.

Thus, it can be expected that bleaching of darker teeth would result in more observable aesthetic colour changes both initially and at the six month recall.

\section{Acknowledgements}

Financial support was obtained from the University of the Western Cape and the DDF fund of the South African Dental Association.

\section{References}

1. Hattab FN, Qudeimat MA, al-Rimawi HS. Dental 1. Discolouration: an overview. J Esthet Dent 1999;11:291-310.

2. Watts A, Addy M. Tooth discolouration and staining: a review of the literature. Br Dent J 2001;190:309-15. 134.

3. Basson R, Grobler SR, vW Kotze TJ, Osman Y. Guidelines for the selection of tooth whitening products amongst those available on the market. SADJ (2013), 68, 122-9.

4. Perchyonok VT, Grobler SR. Tooth-bleaching: mechanism, biological aspects and antioxidants. Int J Dent Oral HIth 2015; 1: doi http://dx.doi.org/10.16966/2378-7090.116.

5. Minolta, Precise colour communication, Minolta, Co., Ltd., Osaka, Japan, 1994; 9242-4830-92 IHCAJ.

6. Grobler SR, Osman Y. Insights into the clinical effectiveness of whitening products - Part 1: Dentist-supervised-at-home bleaching product SADJ 2017;71:363.

7. Grobler SR, Osman Y. Insights into the clinical effectiveness of whitening products. Part 2. Dentist-supervised-at-home LED gel bleaching product. SADJ 2017;72:30-1.

8. Kuehni RG, Marcus RT. An experiment in visual scaling of small color differences. Color Research and Application 1979;4:83-91. 